\title{
Platelet-Rich Plasma and Bone Marrow-Derived Mesenchymal Stromal Cells Prevent TGF- $\beta 1$-Induced Myofibroblast Generation but Are Not Synergistic when Combined: Morphological in vitro Analysis
}

\author{
Flaminia Chellini $^{\mathrm{a}}$ Alessia Tani $^{\mathrm{a}}$ Larissa Vallone $^{\mathrm{a}}$ Daniele Nosi $^{\mathrm{a}}$ Paola Pavan $^{\mathrm{b}}$ \\ Franco Bambi $^{b}$ Sandra Zecchi-Orlandini ${ }^{a}$ Chiara Sassoli ${ }^{a}$ \\ ${ }^{a}$ Department of Experimental and Clinical Medicine, Section of Anatomy and Histology, University of Florence, \\ Florence, Italy; ${ }^{\text {b} T r a n s f u s i o n ~ M e d i c i n e ~ a n d ~ C e l l ~ T h e r a p y ~ U n i t, ~ " A . ~ M e y e r " ~ U n i v e r s i t y ~ C h i l d r e n ' s ~ H o s p i t a l, ~}$ \\ Florence, Italy
}

\section{Keywords}

Fibrosis · Immunofluorescence analysis · Mesenchymal stromal cells $\cdot$ Myofibroblasts $\cdot$ Platelet-rich plasma

\begin{abstract}
The persistence of activated myofibroblasts is a hallmark of fibrosis of many organs. Thus, the modulation of the generation/functionality of these cells may represent a strategical anti-fibrotic therapeutic option. Bone marrow-derived mesenchymal stromal cell (MSC)-based therapy has shown promising clues, but some criticisms still limit the clinical use of these cells, including the need to avoid xenogeneic compound contamination for ex vivo cell amplification and the identification of appropriate growth factors acting as a preconditioning agent and/or cell delivery vehicle during transplantation, thus enabling the improvement of cell survival in the host tissue microenvironment. Many studies have demonstrated the ability of platelet-rich plasma (PRP), a source of many biologically active molecules, to positively influence MSC proliferation, survival, and functionality, as well as its anti-fibrotic potential. Here we investigated the effects of
\end{abstract}

(C) 2019 S. Karger AG, Basel

E-Mail karger@karger.com www.karger.com/cto
PRP, murine and human bone marrow-derived MSCs, and of the combined treatment PRP/MSCs on in vitro differentiation of murine $\mathrm{NIH} / 3 \mathrm{~T} 3$ and human HDFa fibroblasts to myofibroblasts induced by transforming growth factor (TGF)- $\beta 1$, a well-known pro-fibrotic agent. The myofibroblastic phenotype was evaluated morphologically (cell shape and actin cytoskeleton assembly) and immunocytochemically (vinculinrich focal adhesion clustering, a-smooth muscle actin and type- 1 collagen expression). We found that PRP and MSCs, both as single treatments and in combination, were able to prevent the TGF- $\beta 1$-induced fibroblast-myofibroblast transition. Unexpectedly, the combination PRP/MSCs had no synergistic effects. In conclusion, within the limitations related to an in vitro experimentation, our study may contribute to providing an experimental background for supporting the anti-fibrotic potential of the combination PRP/MSCs which, once translated "from bench to bedside," could potentially offer advantages over the single treatments.

(c) 2019 S. Karger AG, Basel

F.C. and A.T. contributed equally to this work
Chiara Sassoli

Department of Experimental and Clinical Medicine, Section of Anatomy and Histology University of Florence, Largo Brambilla 3 IT-50134 Florence (Italy)

E-Mail chiara.sassoli@ unifi.it 


\section{Introduction}

Myofibroblast generation represents a key step of the physiological reparative response to tissue damage in most organs. Myofibroblasts derive mainly, among other potential progenitors, from the differentiation of resident fibroblasts in the extracellular matrix (ECM). Such an event is promoted by the action of several soluble profibrogenic mediators, such as transforming growth factor (TGF)- $\beta 1$ released by infiltrating inflammatory cells (macrophages preferentially) and other local cell types (including the same fibroblasts and myofibroblasts) at the site of the injury, integrated with mechanical stimuli coming from the damaged microenvironment [Pakshir and Hinz, 2018; Weiskirchen et al., 2019]. Myofibroblasts exhibit the features of contractile fibroblasts able to produce and deposit abundant ECM components while transferring mechanical forces to the surrounding ECM. They are characterized by bundles of well-organized actin/myosin-containing microfilaments (stress fibers) connected with extracellular fibronectin filaments through large focal adhesion plaques (fibronexus), by the de novo expression of $\alpha$-smooth muscle actin (sma), the actin isoform found in smooth muscle cells which confers a high contractility to myofibroblasts, and by an abundant rough endoplasmic reticulum, typical of collagensynthetically active fibroblasts. However, when compared to fibroblasts, differentiated myofibroblasts secrete higher amounts of type- 1 and type- 3 collagen, proteoglycans, and other ECM components [Pakshir and Hinz, 2018]. Thus, given these morpho-functional features, in case of damage/wound, myofibroblasts represent the major contributors to the formation of a provisional contractile scar, which enables the reduction of wound size and eventually its closure, required to rapidly restore tissue integrity and preserve organ function. Usually, the healing process proceeds with the remodeling and degradation of the transient scar which is replaced by normal tissue via the accomplishment of regeneration mechanisms. Scar removal is mainly dependent on the balanced and finely tuned activity of proteolytic enzymes selectively digesting individual components of ECM (matrix metalloproteinases, MMPs), and their specific tissue inhibitors (TIMPs), mainly secreted by myofibroblasts. With the end of the healing process, once the physiological organ architecture and function are restored, the number and functionality of myofibroblasts drastically decrease as a result of apoptosis and/or senescence or the possible return to a quiescent state [Jun and Lau, 2018]. In other words, in physiological tissues' reparative acute response to dam- age, the function of myofibroblasts is temporally and spatially limited. By contrast, the permanence and accumulation of myofibroblasts in their activated state has been observed in the case of a persistent or extended damage or in the presence of a persevering inflammatory stimulus, as an aberrant reparative response, as well as in some fibrotic disorders (including systemic fibrotic diseases such as systemic sclerosis, sclerodermatous graft versus host disease and nephrogenic systemic fibrosis, and many organ-specific disorders, such as radiation-induced fibrosis and cardiac, pulmonary, liver, and kidney fibrotic syndromes), whose causative mechanisms are remarkably heterogeneous and, in several instances, even elusive. Under these conditions, myofibroblasts deposit an uncontrolled, excessive, and progressive amount of ECM proteins, which accumulate replacing the normal tissue, thus leading to the disintegration of the organ architecture and, eventually, the loss of its function [Rosenbloom et al., 2017; Weiskirchen et al., 2019]. Therefore, the modulation of the generation, lifespan, and functionality of myofibroblasts may represent a strategical therapeutic option to counteract the progression and, potentially, revert an established fibrosis. Among different potential treatments proposed, bone marrow-derived mesenchymal stromal cell (MSC)-based therapy has shown promising clues. Indeed, accumulating pre-clinical studies in animal models replicating wound healing or fibrotic diseases in different organs have demonstrated the beneficial effects of the administration of these cells, in terms of remarkable attenuation of collagen accumulation and mitigation of scarring [Formigli et al., 2015; Huang et al., 2015; Royce et al., 2015; Srour and Thébaud, 2015; Prockop, 2016; Miao et al., 2017; Kim et al., 2018; Lei et al., 2018; Rozier et al., 2018; Saberi et al., 2019; Xing et al., 2019; Zhao et al., 2019a]. At the same time, in vitro investigations have identified myofibroblast generation as the target of the potential anti-fibrotic paracrine and juxtacrine action of bone marrow MSCs [Galie and Stegemann, 2014; Sassoli et al., 2014b; Huang et al., 2015; Lan et al., 2015; Jang et al., 2015; Fang et al., 2016]. Eventually, some human trials aimed to evaluate bone marrow MSC-based therapy for those diseases where fibrosis plays a major etiological role and are ongoing [Srour and Thébaud, 2015; Squillaro et al., 2016; Lim et al., 2017; Miao et al., 2017; Rozier et al., 2018]. However, despite this encouraging evidence, some criticisms still exist regarding bone marrow MSC-based therapy, limiting their use in clinical applications including, among others: (i) the need of protocols for ex vivo cell amplification according to GMP guidelines, avoiding the contamination or immunologi- 
cal reactions towards xenogeneic compounds, such as animal sera, and (ii) the identification of appropriate growth factors and/or bioengineered three-dimensional scaffolds acting both as a pre-conditioning agent and cell delivery vehicle during transplantation, thus enabling to preserve or promote the survival rate, optimize functionality, and improve the engraftment of the injected cells in the host tissue microenvironment. Indeed, such a microenvironment is often characterized by nutrient deprivation and reduced oxygen tension that greatly reduces the vitality of bone marrow MSCs, thus limiting their therapeutic potential [Andia et al., 2018; Hu and Li, 2018; Ferro et al., 2019]. In this context, advantages could come from the use of platelet-rich plasma (PRP), a plasma fraction with a concentration of platelets above baseline levels, easily available from whole blood of patients and representing a cost-effective reservoir of many biologically active molecules. In fact, many studies have demonstrated the ability of this blood product to improve the proliferation, survival, and functionality of bone marrow-derived MSCs [Formigli et al., 2012; Amable et al., 2014; Rubio-Azpeitia and Andia, 2014; Jalowiec et al., 2016; Hosni Ahmed et al., 2017; Hoberman et al., 2018; Sassoli et al., 2018b]. Moreover, the ability of PRP to limit fibrosis in different damaged and/or diseased organs has been observed [Cianforlini et al., 2015; Vu et al., 2015; Moghadam et al., 2017; Jang et al., 2017; Sanchez-Avila et al., 2018; Sayadi et al., 2018; Shoeib et al., 2018; Tavukcu et al., 2018] and experimental evidence suggests that antifibrotic potential of PRP may be correlated to its ability to prevent myofibroblast generation [Anitua et al., 2012; Anitua et al., 2015; Chellini et al., 2018]. On such premises, the present in vitro study intended to investigate the effects of a combined treatment with bone marrow-derived MSCs and PRP on TGF- $\beta 1$-induced myofibroblast generation.

\section{Materials and Methods}

\section{Cell Cultures and Treatments}

Murine NIH/3T3 fibroblasts from American Type Culture Collection (ATCC, Manassas, VA, USA), and human dermal fibroblast cells - HDFa - from ScienCell (Carlsbad, CA, USA) were grown in proliferation medium (PM) containing DMEM supplemented with $10 \%$ fetal bovine serum (FBS) and $1 \%$ penicillin/ streptomycin (Sigma, Milan, Italy) at $37^{\circ} \mathrm{C}$ in a humidified atmosphere of $5 \% \mathrm{CO}_{2}$. These cells served as undifferentiated cells. Fibroblasts were induced to differentiate towards myofibroblasts by culturing in differentiation medium (DM) containing a low serum concentration ( $2 \% \mathrm{FBS}$, Sigma) and the well-known pro-fibrotic agent TGF- $\beta 1$ ( 2 ng/mL, PeproTech Inc., Rocky Hill, NJ, USA) for
$48 \mathrm{~h}$ or 5 days as previously reported [Chellini et al., 2018]. These cells served as differentiated control cells. In parallel experiments, in order to evaluate the effects of PRP and/or bone marrow-derived MSCs on fibroblast-myofibroblast transition, both murine and human fibroblasts were cultured in the presence of PRP added to $\mathrm{DM}$ at the dilution of 1:50 (DM + PRP) or co-cultured with murine or human adult bone marrow-derived MSCs in DM, in the absence or presence of PRP (1:50) for $48 \mathrm{~h}$ and 5 days. Murine and human MSCs were previously collected from the bone marrow of femora and tibiae of male C2F1 mice and from the iliac crest and aspirates of normal human donors, respectively, immunophenotypically and morphologically in vitro characterized, cryopreserved in a solution containing 10\% dimethyl sulfoxide and $90 \%$ FBS, in a liquid nitrogen tank [Sassoli et al., 2018b; Tani et al., 2018] and used after proper thawing for the present experimentation. The thawed cells were cultured in their specific PM, containing DMEM for murine MSCs or DMEM plus GlutaMAX ${ }^{\mathrm{TM}}$ for human MSCs, supplemented with $20 \%$ FBS, and $1 \%$ penicillin/streptomycin (Sigma).

Before seeding in the co-culture with fibroblasts (ratio fibroblasts/MSCs, 1:2) in order to distinguish the two cell types, MSCs were labeled with the fluorescent Vybrant ${ }^{\mathrm{TM}}$ Dil Cell-Labeling solution (Dil-MSCs) according to the manufacturer's instructions (Molecular Probes, Eugene, OR, USA), as reported previously [Sassoli et al., 2018b].

\section{PRP Preparation}

Ready-to-use thawed PRP aliquots previously prepared and stored at $-80{ }^{\circ} \mathrm{C}$ [Chellini et al., 2018] were used for the present experiments. Briefly, PRP was obtained from adult healthy donors undergoing a plasma-platelet apheresis (Haemonetics $\mathrm{MCS}^{\circledR}$, Haemonetics, Milan, Italy), after receiving proper written informed consent. Collected platelet units were stored in a specific shaker incubator and the plasma ones were immediately frozen at $-80^{\circ} \mathrm{C}$ and subsequently thawed at $4^{\circ} \mathrm{C}$ for $16 \mathrm{~h}$ to obtain the cryoprecipitate by syphoning. Successively, platelets were resuspended in cryoprecipitate and adjusted to a final concentration of $2 \times 10^{6} /$ $\mu \mathrm{L}$. Platelet activation was induced by the addition of a calcium digluconate solution (10\%). After being tested for microbiological contamination and classified as not suitable for transfusion-infusion purposes, ready-to-use PRP aliquots were stored at $-80^{\circ} \mathrm{C}$. The use of PRP in experimental in vitro protocols does not require Ethical Committee approval.

\section{Fluorescent Plasma Membrane and F-Actin Labeling}

$\mathrm{NIH} / 3 \mathrm{~T} 3$ fibroblasts in monoculture or in co-culture with DilMSCs in the different experimental conditions, seeded on glass coverslips, were fixed with $0.5 \%$ paraformaldehyde (PFA) in PBS for $10 \mathrm{~min}$ at room temperature (RT). After washing, the cells were incubated with Alexa Fluor 488-conjugated wheat germ agglutinin (WGA, 1:100; Molecular Probes) for $10 \mathrm{~min}$ at RT which binds glycoconjugates, i.e., $\mathrm{N}$-acetylglucosamine and $\mathrm{N}$-acetylneuraminic acid (sialic acid) residues, on cell membranes. In parallel experiments fixed cells were permeabilized with cold acetone for $3 \mathrm{~min}$, incubated with a blocking solution consisting of $1 \%$ bovine serum albumin (BSA; Sigma) in PBS for 40 min and then with Alexa Fluor 488-labeled phalloidin (1:40; Molecular Probes) for $20 \mathrm{~min}$ at RT to detect actin filament organization (F-actin) prior to being immunolabeled for vinculin expression evaluation. After washing, the coverslips containing the stained cells were 

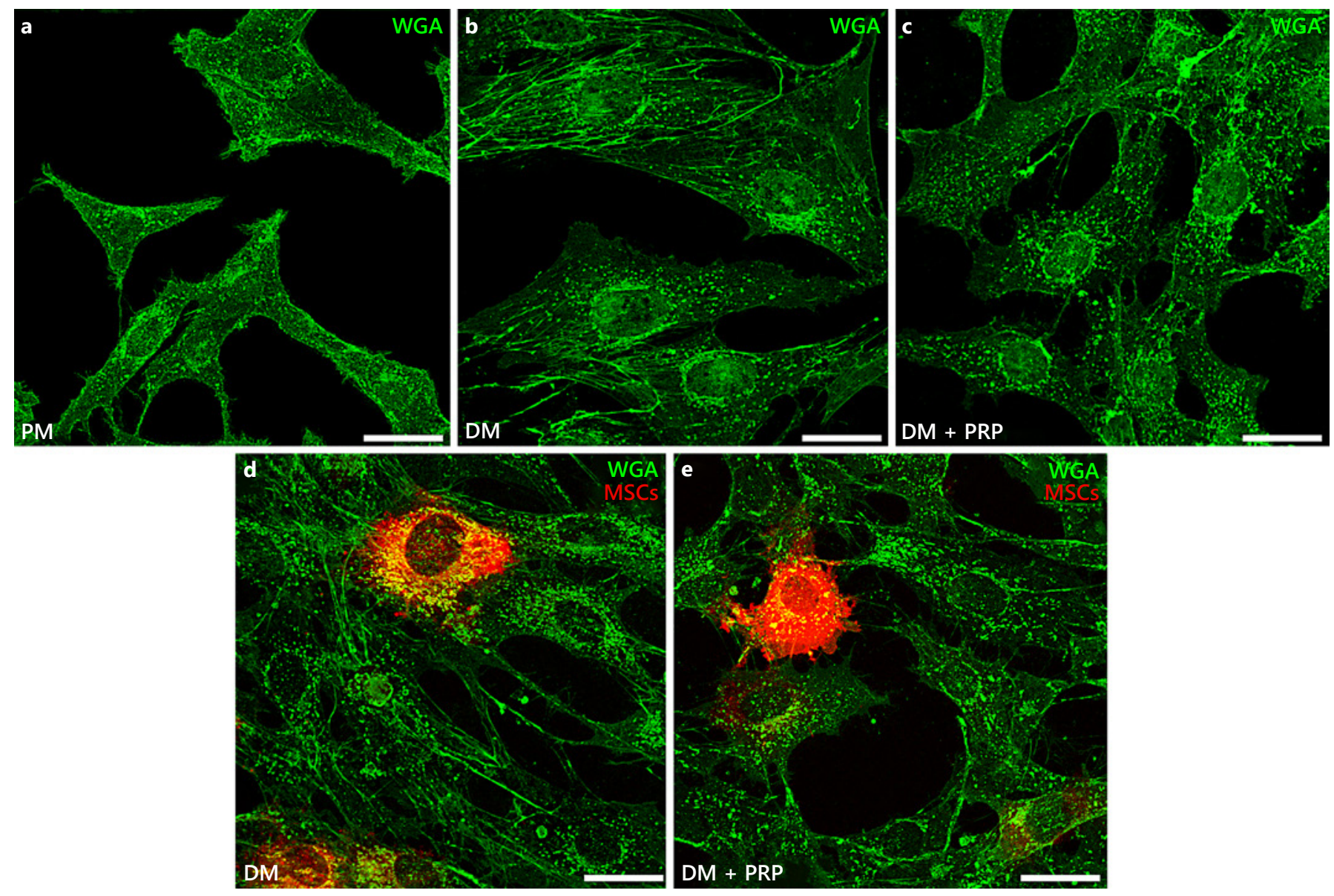

Fig. 1. Morphological evaluation of the fibroblast/myofibroblast phenotype. Representative images of fixed murine NIH/3T3 fibroblasts in monoculture $(\mathbf{a}-\mathbf{c})$ or in co-culture $(\mathbf{d}, \mathbf{e})$ with Dil-labeled

murine MSCs (red) in the indicated experimental conditions stained with Alexa Fluor 488-conjugated WGA (green) to reveal the plasma membrane. Scale bar, $30 \mu \mathrm{m}$.

mounted with an antifade mounting medium (Biomeda Gel Mount, Electron Microscopy Sciences, Foster City, CA, USA) and observed under a confocal Leica TCS SP5 microscope equipped with a $\mathrm{HeNe} / \mathrm{Ar}$ laser source for fluorescence measurements (Leica Microsystems, Mannheim, Germany). Observations were performed using a Leica Plan Apo 63×/1.43NA oil immersion objective. A series of optical sections $(1,024 \times 1,204$ pixels each; pixel size $204.3 \mathrm{~nm}) 0.4 \mu \mathrm{m}$ in thickness were taken throughout the depth of the cells at intervals of $0.4 \mu \mathrm{m}$. Images were then projected onto a single "extended focus" image. Densitometric analysis of the intensity of F-actin was performed on digitized images using ImageJ $1.49 \mathrm{v}$ software (http://rsbweb.nih.gov/ij) in 20 regions of interest (ROI) of $100 \mu \mathrm{m}^{2}$ for each confocal stack (at least 10).

\section{Confocal Immunofluorescence}

Fibroblasts in monoculture or in co-culture with Dil-MSCs seeded on glass coverslips in the different experimental conditions were fixed with $0.5 \%$ PFA in PBS for 10 min at RT. After permeabilization with cold acetone for 3 min and blocking with a solution of $0.5 \%$ BSA (Sigma) and 3\% glycerol in PBS for 20 min, fixed cells were incubated overnight at $4{ }^{\circ} \mathrm{C}$ with the following antibodies: mouse monoclonal anti-vinculin (1:100; Sigma), mouse monoclonal anti- $\alpha$-sma (1:100; Abcam, Cambridge, UK), and rabbit polyclonal anti-type-1 collagen (1:50; Santa Cruz Biotechnology, Santa Cruz, CA, USA). The immunoreactions were revealed by incubation with specific anti-mouse Alexa Fluor 488- or Cy5-conjugated IgG or anti-rabbit Alexa Fluor 488-conjugated IgG (1:200; Molecular Probes) for $1 \mathrm{~h}$ at RT. Negative controls were carried out by replacing the primary antibodies with non-immune serum; crossreactivity of the secondary antibodies was tested in control experiments in which primary antibodies were omitted. After washing, the coverslips containing the immunolabeled cells were mounted with an antifade mounting medium (Biomeda Gel Mount) and observed under a confocal Leica TCS SP5 microscope as reported in the previous paragraph. Densitometric analyses of the intensity of vinculin, $\alpha$-sma, and type- 1 collagen fluorescence signals were performed on digitized images using ImageJ 1.49v software (http:// rsbweb.nih.gov/ij) in $20 \mathrm{ROI}$ of $100 \mu \mathrm{m}^{2}$ for each confocal stack (at least 10). 


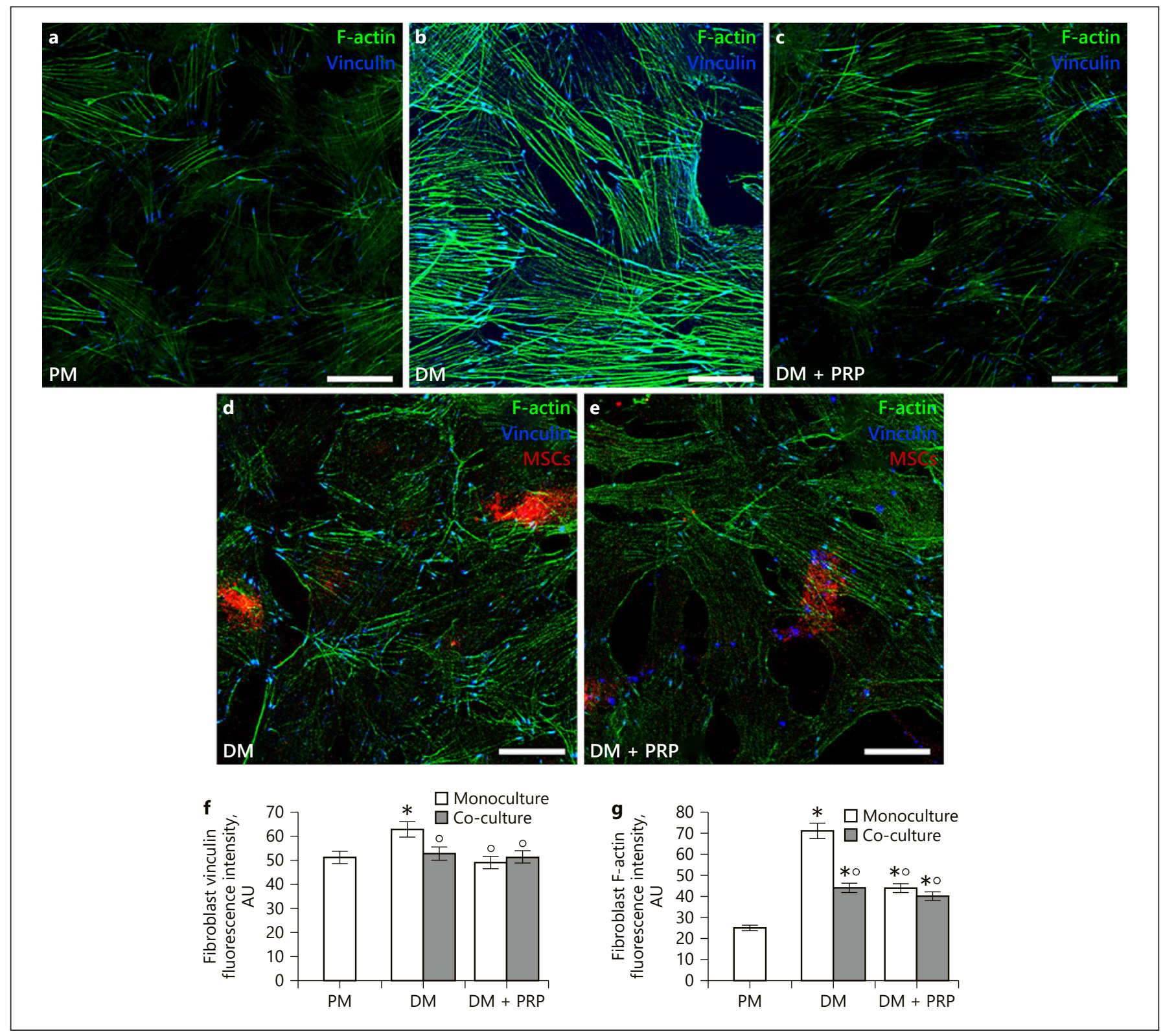

Fig. 2. Evaluation of murine NIH/3T3 fibroblast to myofibroblast conversion: cytoskeleton organization. Representative confocal fluorescence images of fibroblasts in monoculture (a-c) or in coculture (d, e) with Dil-labeled murine MSCs (red) in the indicated experimental conditions stained with Alexa Fluor 488-conjugated phalloidin to reveal F-actin (green) and immunostained with an-

\section{Statistical Analysis}

Data are presented as the mean \pm standard error of the mean (SEM) as results of at least three independent experiments performed in triplicate and analyzed using one-way ANOVA with post hoc Tukey HSD. Differences were considered significant at $p<0.05$. Calculations were performed using GraphPad Prism 4.0 statistical software (GraphPad, San Diego, CA, USA).

PRP and MSCs Prevent FibroblastMyofibroblast Transition tibodies against vinculin (cyan). Scale bar, $30 \mu \mathrm{m}$. Histograms showing the densitometric analysis of the intensity of vinculin (f) and F-actin (g) fluorescence signals, performed on digitized images. AU, arbitrary units. Significance of differences: ${ }^{*} p<0.05$ vs. $\mathrm{PM} ;{ }^{\circ} p<0.05$ vs. DM monoculture.

\section{Results}

The myofibroblastic phenotype was evaluated morphologically and immunocytochemically. We found that control differentiated murine $\mathrm{NIH} / 3 \mathrm{~T} 3$ fibroblasts cul- 

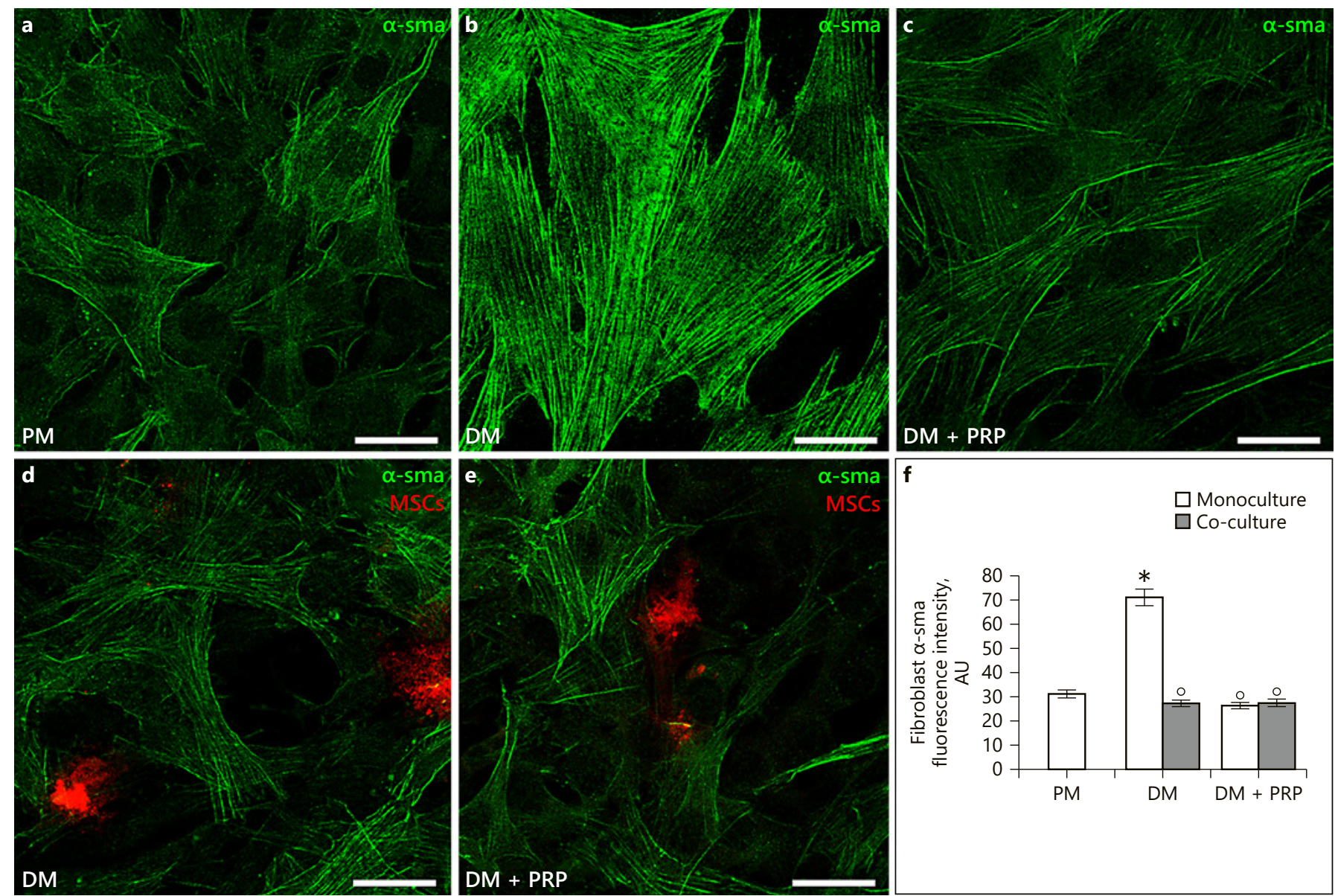

Fig. 3. Evaluation of murine NIH/3T3 fibroblast to myofibroblast conversion: $a$-sma expression. Representative confocal fluorescence images of fibroblasts in monoculture $(\mathbf{a}-\mathbf{c})$ or in co-culture (d, e) with Dil-labeled murine MSCs (red) in the indicated experimental conditions immunostained with antibodies against $\alpha$-sma

(green). Scale bar, $30 \mu \mathrm{m}$. $\mathbf{f}$ Histogram showing the densitometric analysis of the intensity of the $\alpha$-sma fluorescence signal, performed on digitized images. AU, arbitrary units. Significance of differences: ${ }^{*} p<0.05$ vs. $\mathrm{PM} ;{ }^{\circ} p<0.05$ vs. DM monoculture.

tured in DM for $48 \mathrm{~h}$ exhibited the typical features of myofibroblasts (Fig. 1). Indeed, they appeared much larger with a more polygonal shape as compared to the undifferentiated cells cultured in PM which, instead, were smaller and spindle shaped as judged by the confocal fluorescence analysis of the cells labeled with the membrane dye Alexa Fluor 488-conjugated WGA (Fig. 1a, b). The analysis of cytoskeleton organization (Fig. 2) revealed that the differentiated cells, consistent with their shape, possessed prominent and parallely organized bundles of F-actin filaments, extending through the length of the cells, most likely stress fibers, and a strong immunoreactivity for vinculin, which appeared mainly aggregated in large complexes attached at the ends of the actin filaments (Fig. 2b, f, g). By contrast, undifferentiated cells exhibited thin and less organized F-actin filaments and a low ex-

pression of vinculin accumulating in small dot-like aggregates at either the cell border or scattered throughout the cytoplasm (Fig. 2a, f, g). Contrary to undifferentiated cells, the murine cells cultured in DM for $48 \mathrm{~h}$ showed a robust expression of $\alpha$-sma, the most reliable marker of myofibroblasts, which seems to coincide with stress fibers (Fig. 3a, b, f). The cells, after 5 days in DM, showed an increase in the expression of type-1 collagen at the cytoplasmic level, with a distinctive pattern of distribution consistent with a localization of the protein in the cisternae of the endoplasmic reticulum and Golgi vesicles, suggestive for a high cellular protein synthetic activity, a distinctive feature, likely discriminating fibroblasts from myofibroblasts (Fig. 4a, b, f). Moreover in some cases, positivity for type-1 collagen was found outside the cells in a filamentous form (Fig. 4b). 

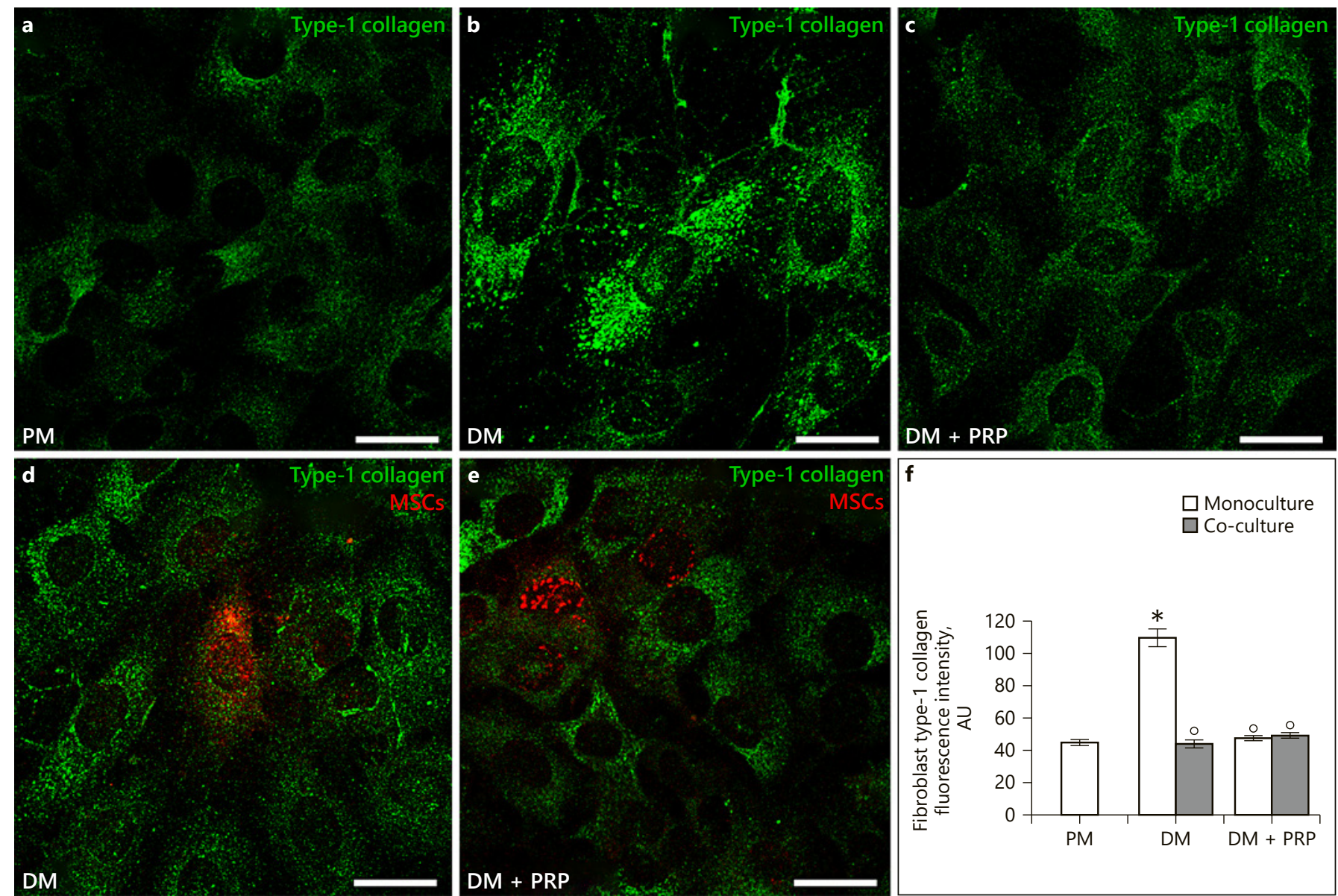

Fig. 4. Evaluation of murine NIH/3T3 fibroblast to myofibroblast conversion: type-1 collagen expression. Representative confocal fluorescence images of fibroblasts in monoculture (a-c) or in coculture (d, e) with Dil-labeled murine MSCs (red) in the indicated experimental conditions immunostained with antibodies against type- 1 collagen (green). Scale bar, $25 \mu \mathrm{m}$. $\mathbf{f}$ Histogram showing the densitometric analysis of the intensity of the type- 1 collagen fluorescence signal, performed on digitized images. AU, arbitrary units. Significance of differences: ${ }^{*} p<0.05$ vs. PM; ${ }^{\circ} p<0.05$ vs. DM monoculture.
When murine fibroblasts were cultured in DM in the presence of PRP (DM + PRP) they exhibited a different morphology with respect to differentiated cells in DM, more similar to that of cells cultured in PM (Fig. 1a-c), together with a marked reduction of the assembly of stress fibers and the expression and organization of vinculin at focal adhesion sites (Fig. 2a-c, f, g), a strong decrease of $a$-sma (Fig. 3a-c, f) and type- 1 collagen (Fig. $4 a-c, f$ ) expression, thus supporting the ability of PRP to counteract the fibroblast-myofibroblast transition promoted by TGF- $\beta 1$. Morphological and immunophenotypical features similar to those displayed by DM + PRP-treated cells were observed in murine fibroblasts co-cultured with murine MSCs in DM (Fig. 1c, d, Fig. 2c, d, f, g, Fig. 3c, d, f, Fig. 4c, d, f), suggesting the ability of juxtacrine-paracrine factors from MSCs to negatively interfere with the
TGF- $\beta 1$ signaling pathway in terms of fibroblast myodifferentiation.

Of note, the combination PRP + MSCs did not elicit synergistic effects: indeed, murine fibroblasts co-cultured with MSCs in DM in the presence of PRP behaved as fibroblasts cultured in the presence of PRP or co-cultured with MSCs showing a comparable morphology (Fig. 1ce) and no significant differences in terms of F-actin (Fig. 2c-e, g), vinculin (Fig. 2c-f), $\alpha$-sma (Fig. 3c-f), and type-1 collagen (Fig. 4c-f) expression.

Likewise, primary human dermal fibroblasts ( $\mathrm{HDF} \alpha)$ cultured in DM + PRP or co-cultured in DM with human MSCs in the absence or presence of PRP appeared more spindle shaped with reduced expression levels of $\alpha$-sma, which also appeared less organized along filaments, as compared to differentiating control cells cultured in DM 

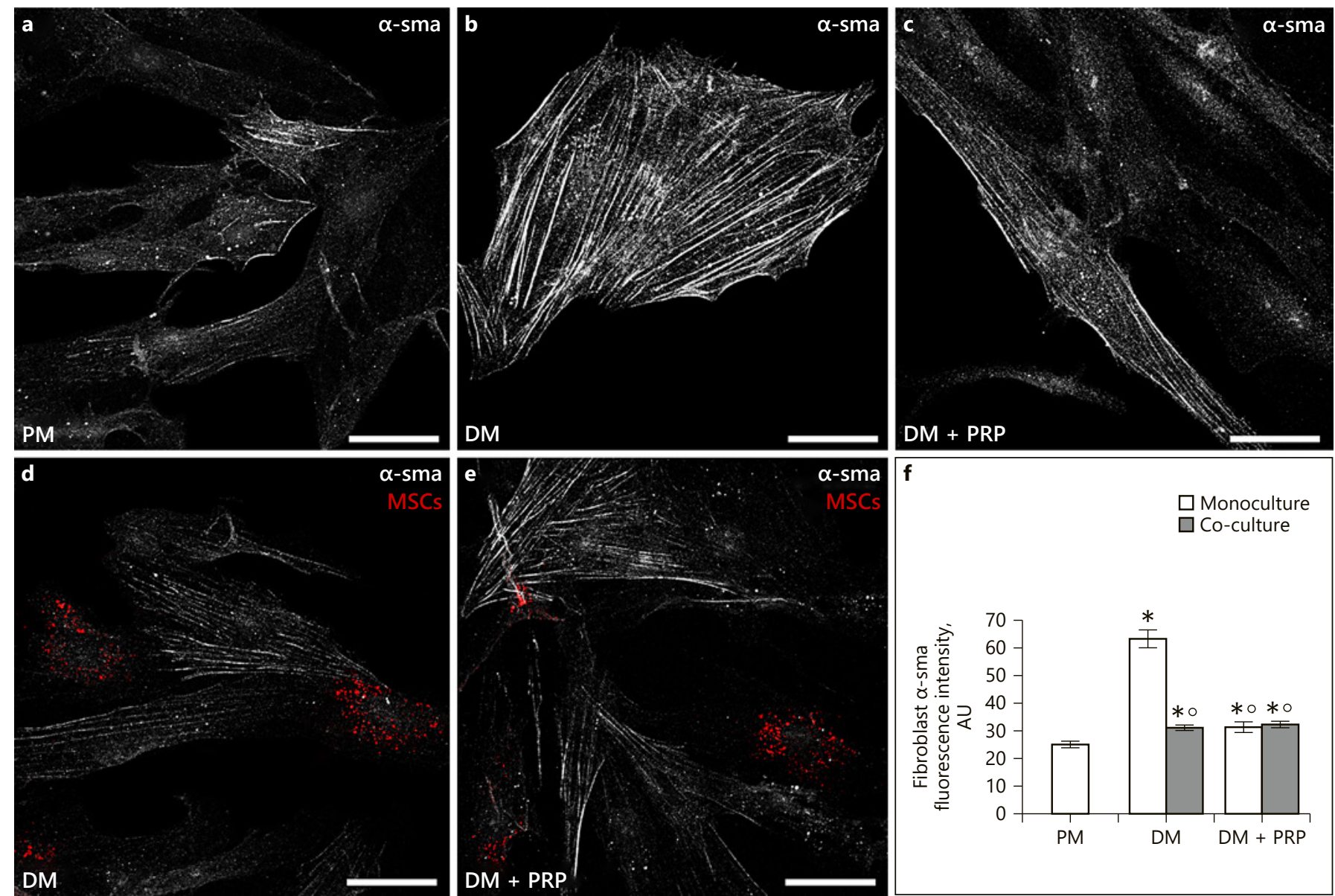

Fig. 5. Evaluation of human $\mathrm{HDF}$ a fibroblast to myofibroblast conversion. Representative confocal fluorescence images of fibroblasts in monoculture (a-c) or in co-culture $(\mathbf{d}, \mathbf{e})$ with Dil-labeled murine MSCs (red) in the indicated experimental conditions immunostained with antibodies against $\alpha$-sma (grey). Scale bar, 50

$\mu \mathrm{m}$. f Histogram showing the densitometric analysis of the intensity of the $\alpha$-sma fluorescence signal, performed on digitized images. AU, arbitrary units. Significance of differences: ${ }^{*} p<0.05$ vs. $\mathrm{PM} ;{ }^{\circ} p<0.05$ vs. DM monoculture.

(Fig. 5). It must be pointed out that MSCs in co-culture with either murine and human fibroblasts, both in the absence or presence of PRP, failed to express $\alpha$-sma and displayed very low levels of type-1 collagen expression, indicating that, at least in our experimental conditions, these cells were unable to differentiate towards myofibroblasts.

\section{Discussion}

The identification of effective therapeutic options aimed to prevent and even more counteract and revert excessive tissue scarring is a current scientific challenge with high clinical priority. This is on the basis of the following considerations: (i) fibrosis frequently occurs in different organs as an aberrant maladaptive reparative re- sponse to extended/severe or chronic injury, often leading to their morpho-functional impairment and endstage organ disease; (ii) therapeutic tools for eradication of the underlying etiology (in some cases resulting in fibrosis resolution) are not always available and, even more, most human fibrotic diseases are often multifactorial in origin, so that a direct action on the noxious causes may be virtually impossible; (iii) current anti-fibrotic therapeutic options adopted so far have a limited efficacy; (iv) fibrosis is estimated to account for up to $50 \%$ of all causes of death in industrialized countries [Fang et al., 2017; Lim et al., 2017; Zuccaro et al., 2017; Allinovi et al., 2018; Walraven et al., 2018; Yoon et al., 2019]. In this scenario, therapies aimed to modulate the recognized "core cellular mechanisms of fibrosis" such as generation, functionality, and fate of myofibroblasts, the key effectors of 
tissue scarring in most organs, could be strategical and promising. In such a view the findings of the present study, showing that PRP and bone marrow-derived MSCs, both as single treatments and in combination, are able to inhibit the TGF- $\beta 1$-induced conversion of fibroblasts in myofibroblasts, are of particular interest, contributing to design potential effective anti-fibrotic options. Data related to PRP used as a single treatment essentially confirmed previous research [Anitua et al., 2012, 2015] and our recent study demonstrating that PRP, acting through vascular endothelial growth factor (VEGF)A/VEGF receptor-1(VEGFR-1 or Flt-1)-mediated signaling to antagonize TGF- $\beta 1 / \mathrm{Smad} 3$ signaling, prevented fibroblast-myofibroblast transition, and did not induce per se fibroblast myodifferentiation [Chellini et al., 2018]. The data of the co-culture of murine fibroblasts and bone marrow-derived MSCs in DM (i.e., in the presence of the profibrotic agent TGF- $\beta 1$ ) in the absence of PRP, consistent with the findings from other research groups [Galie and Stegemann, 2014; Huang et al., 2015; Jang et al., 2015; Lan et al., 2015; Fang et al., 2016], extended our previous research aimed to define the role of bone marrow-derived MSCs on the regulation of MMPs in skeletal muscle cells including fibroblasts [Sassoli et al., 2014b]. In particular in that report we showed that murine bone marrow-derived MSCs induced an upregulation of MMP-2 and MMP-9 expression, concomitantly to a reduction of TIMP-1 and TIMP-2 in murine primary skeletal fibroblasts and inhibited their myofibroblastic differentiation, induced by a low serum culture condition, by means of juxtacrine and paracrine mechanisms. In addition, in the same paper, the pharmacological inhibition of MMPs prevented in these cells, the decrease of $\alpha$-sma and type- 1 collagen expression induced by MSCs, suggesting that MSCs could attenuate the fibrogenic response via mechanisms mediated by MMPs. In the present study, we showed the capability of bone marrow-derived MSCs to negatively interfere with fibroblast/myofibroblast transition induced by TGF- $\beta 1$, both in murine and human coculture cell systems. Experiments are ongoing in our lab to identify the potential molecular mechanisms underpinning the fibroblasts' response to MSCs. However, an involvement of VEGF-A can be postulated taking into consideration that VEGF-A is released by bone marrowderived MSCs [Sassoli et al., 2012] and that the role of VEGF-A in inhibiting myofibroblast differentiation, independently, or at least in part independently, from its ability to negatively regulate TGF- $\beta 1$ signaling, has been demonstrated [Chellini et al., 2018]. On the other hand, other paracrine factors of MSC secretome may negatively

PRP and MSCs Prevent FibroblastMyofibroblast Transition interfere with TGF- $\beta 1$ signaling, synergistically acting as anti-fibrotic agents such as basic fibroblast growth factor, interleukin-15 and interferon-gamma [Sassoli et al., 2012; Yang et al., 2016; Gallego-Muñoz et al., 2017; Manohar et al., 2018]. Moreover, we have recently demonstrated the expression and release of functional MMP-2 by bone marrow MSCs and their autocrine regulation by the sphingosine 1-phosphate $(\mathrm{S} 1 \mathrm{P}) / \mathrm{S} 1 \mathrm{P}$ receptor axis [Sassoli et al., 2014a, 2018a]. When considering the evidence of an interaction between MMPs (including MMP-2) and syndecans [Manon-Jensen et al., 2013], proven to be profibrotic molecules along the TGF- $\beta 1$ axis [Lunde et al., 2016], we can also speculate that bone marrow MSCs could exert their inhibitory effects on fibroblast-myofibroblast conversion via MMP-2. Furthermore, the involvement of juxtacrine mechanisms including the activation of the Notch-1-mediated pathway needs to be investigated, in view of the known ability of this pathway to negatively modulate fibroblast-myofibroblast transition by downregulating TGF- $\beta 1 / \mathrm{Smad} 3$ signaling [Sassoli et al., 2013, Nistri et al., 2017] and given that bone marrow MSCs may activate this pathway by providing Notch-1 ligands such as Jagged-1 [Sassoli et al., 2011; Duryagina et al., 2013]. Notably, differently from what was expected, the effects elicited by PRP and MSCs when used in combination were not additive or synergistic, but almost superimposable to those elicited by the single treatments.

We expected that the combination PRP/MSCs could amplify the effects of the single treatments and more efficiently counteract fibroblast myodifferentiation, assuming that the combination could result in an increased concentration of anti-fibrotic factors such as VEGF-A, contained in both PRP [Chellini et al., 2018] and MSC secretome [Sassoli et al., 2012], and that some factors contained in PRP (such as platelet-derived growth factor, PDGF-BB) could stimulate the ability of MSCs to release anti-fibrotic factors (a cross-talk between PDGF-BB and VEGF-A in bone marrow-derived MSCs has been reported) [Xu et al., 2015], possibly negatively interfering with TGF- $\beta$, likely contained in PRP [Qiao et al., 2017].

The lack of additive/synergistic effects of the combined treatment on fibroblasts may be explained by the establishment of more complex mutual paracrine and juxtacrine interactions between fibroblasts and bone marrow MSCs in the presence of PRP that may affect the reciprocal behavior of both cell types, at least in our cell system. For example, PRP-derived molecules may stimulate fibroblasts to release factors which, in turn, may affect the functionality of MSCs, including their secretome, in terms of the ratio between pro- and anti-fibrotic agents. On the other hand, 
we can also postulate that the combined treatment could result in an increase of the levels of pro-fibrotic factors, derived both from PRP and bone marrow MSCs, including S1P [Sassoli et al., 2014a; Hoeferlin et al., 2015; Vestri et al., 2017], capable of activating pro-fibrotic signaling pathways - concomitantly to anti-fibrotic ones - likely not activated by the single treatments. This could be due to the under threshold concentration of the pro-fibrotic agents in the single treatments, not enough to evoke a cell response, also taking into account the dose dependence of some fibroblastic responses to different factors, including S1P [ $\mathrm{Li}$ et al., 2011; Xian et al., 2015; Aoyama-Araki et al., 2017; Duan et al., 2017]. Further comprehensive studies to test all these hypotheses are required. On the other hand, we cannot exclude the possibility that the effects we observed are strictly related to the in vitro experimentation condition that eliminates, among others, many paracrine-juxtacrine mechanisms regulating cell interactions and functionality (e.g., stimuli from blood vessels or immunological controls) as well as mechanical stimuli, normally available in vivo and to the experimental times.

However, the combination PRP/MSCs may offer advantages over the single treatments. In fact, the advantage of an MSC-based therapy approach over the administration of PRP alone may rely on the possibility that metabolically active cells may represent a continuous source of bioactive molecules/anti-fibrotic agents released in the context of damaged tissue, thus potentially assuring a long lasting action. Moreover, it has been proven that many factors are released by the cells within extracellular vesicles/exosomes that preserve such factors from degradation [Zhao et al., 2019b]. On the other hand, since PRP promotes bone marrow MSC viability, survival, and proliferation [Formigli et al., 2012; Amable et al., 2014; Rubio-Azpeitia and Andia, 2014; Jalowiec et al., 2016; Hosni Ahmed et al., 2017; Hoberman et al., 2018; Sassoli et al., 2018b], it may contribute to overcome the most critical challenges associated with the application of bone marrow MSCs in clinical cell therapies, namely the need of cell expansion in culture before transplantation avoiding animal-derived growth factors and sera as well as of preservation and even promotion of engrafted MSC survival and function.

In addition, it is tempting to speculate that PRP may also modulate the functionality of the tissue-resident interstitial MSCs or endogenous MSCs recruited from systemic circulation and migrated at the site of injury, whose role in balancing tissue repair and fibrosis is becoming increasingly relevant [Rabelink and Little, 2013; El Agha et al., 2017]. Last but not least, we have recently demonstrated that the combined treatment of PRP/MSCs was more effective than
PRP alone in stimulating the activation and differentiation along the myogenic program of skeletal muscle progenitors cells (myoblasts/satellite cells), responsible for the formation of new multinucleated myofibers after damage [Sassoli et al., 2018b]. It is well known that a maladaptive fibrotic scar may often occur in response to an extended or severe muscle damage hampering the functionality of satellite cells and eventually the complete tissue morpho-functional recovery of damaged tissue [Chellini et al., 2019; Mahdy, 2019]. From such a view the findings of the present study may suggest that at least the injured skeletal muscle could double benefit from the combined treatment with MSCs and PRP, which may concomitantly contribute to the recreation of an anti-fibrogenic microenvironment, more conductive for muscle progenitor functionality, and to direct stimulation of the endogenous mechanisms of tissue repair/regeneration.

In conclusion, within the limitations related to an in vitro experimentation, our study contributes to provide experimental background for supporting the anti-fibrotic potential of the association of MSCs and PRP. It must, however, be pointed out that many critical issues related to the clinical application of both PRP and MSCs still exist. PRP criticisms are mainly represented by the heterogeneity of formulations, owing to the different non-standardized preparation procedures and by the lack of univocal guidelines for the best usage with respect to dose, optimal timing of administration, frequency and customization for targeted tissues, which may account for the reported conflicting results concerning the effects of this blood product in the modulation of tissue fibrosis [Delos et al., 2014; Cianforlini et al., 2015; Kelc and Vogrin, 2015; Reurink et al., 2015; Vu et al., 2015; Guillodo et al., 2016; Lynch and Bashir, 2016; Schroeder et al., 2016; Moghadam et al., 2017; Jang et al., 2017; Sanchez-Avila et al., 2018; Sayadi et al., 2018; Shoeib et al., 2018; Tavukcu et al., 2018; Chellini et al., 2019]. On the other hand, it is worth reminding that a single biomarker or a reliable combination of biomarkers that specifically and/or exclusively define bone marrow MSCs, are still unidentified, thus leading to the risk of getting a not homogeneous cell culture, even contaminated by non-mesenchymal cells. Moreover, different in vitro culture procedures for cell expansion may affect cell properties, and long-term studies are required to elucidate the side effects as well as to validate the safety and effectiveness of bone marrow MSCbased therapy for anti-fibrotic and pro-regenerative purposes [Sassoli et al., 2012; Li et al., 2016; Prockop, 2016; Squillaro et al., 2016; Andia et al., 2018]. All considered, as always, the translation from bench to bedside has to be evaluated with great caution. 


\section{Acknowledgements}

The research group of Dr. Riccardo Saccardi (Careggi University Hospital, Florence, Italy) is gratefully acknowledged for having kindly provided bone marrow-derived MSCs.

\section{Statement of Ethics}

The experimental protocols including animals were conform to the European Community guidelines for animal care (DL 116/92, application of the European Communities Council Directive of 24 November 1986; 86/609/EEC) and approved by the Committee for Animal Care and Experimental Use of the University of Florence, No. A5278-01. The protocols were communicated to local authorities and to the Italian Ministry of the Health; according to the Italian law (Art.7/D.lgs 116/92) such a procedure does not require Ministry authorization.

PRP was obtained from the whole blood of adult healthy volunteers after receiving written informed consent and provided in ready-to-use aliquots classified as not suitable for transfusion-infusion purposes. Its use in experimental in vitro protocols does not require Ethical Committee approval.

Human bone marrow-derived MSCs were obtained from normal donors from the iliac crest and aspirates. All donors gave their informed consent for inclusion before they participated in the study. The study was conducted in accordance with the Declaration of Helsinki, and the protocol was approved by the local Ethics Committee of the University of Florence, Italy (prot. No. 23/2007).

\section{Disclosure Statement}

The authors have no conflicts of interest to declare.

\section{Funding Sources}

This research was funded by MIUR (Ministero dell'Istruzione dell'Università e della Ricerca - University of Florence, Italy; research funds to D.N., S.Z.-O., and C.S.)

\section{Author Contributions}

Conceptualization, F.C., A.T., and C.S.; formal analysis, D.N.; investigation, F.C., A.T., L.V., and C.S.; resources, D.N., P.P., F.B., S.Z.-O., and C.S.; data curation, F.C., A.T., D.N., and C.S.; writing - original draft preparation, C.S.; writing - review and editing, F.C., A.T., S.Z.-O., and C.S.; visualization, F.C., A.T., D.N., S.Z.-O., and C.S.; funding acquisition, D.N., S.Z.-O., and C.S. All authors approved the final version of the work.

\section{References}

Allinovi M, De Chiara L, Angelotti ML, Becherucci F, Romagnani P. Anti-fibrotic treatments: a review of clinical evidence. Matrix Biol. 2018 Aug;68-69:333-54.

Amable PR, Teixeira MV, Carias RB, Granjeiro JM, Borojevic R. Mesenchymal stromal cell proliferation, gene expression and protein production in human platelet-rich plasma-supplement media. PLoS One. 2014 Aug;9(8):e104662.

Andia I, Martin JI, Maffulli N. Platelet-rich Plasma and Mesenchymal Stem Cells: Exciting, But ... are we there Yet? Sports Med Arthrosc Rev. 2018 Jun;26(2):59-63.

Anitua E, de la Fuente M, Muruzabal F, Riestra A, Merayo-Lloves J, Orive G. Plasma rich in growth factors (PRGF) eye drops stimulates scarless regeneration compared to autologous serum in the ocular surface stromal fibroblasts. Exp Eye Res. 2015 Jun;135:118-26.

Anitua E, Troya M, Orive G. Plasma rich in growth factors promote gingival tissue regeneration by stimulating fibroblast proliferation and migration and by blocking transforming growth factor- $\beta 1$-induced myodifferentiation. J Periodontol. 2012 Aug;83(8):1028-37.

Aoyama-Araki Y, Honjo M, Uchida T, Yamagishi R, Kano K, Aoki J, et al. Sphingosine-1-Phosphate (S1P)-Related Response of Human Conjunctival Fibroblasts After Filtration Surgery for Glaucoma. Invest Ophthalmol Vis Sci. 2017 Apr;58(4):2258-65.
Chellini F, Tani A, Vallone L, Nosi D, Pavan P, Bambi F, et al. Platelet-Rich Plasma Prevents In Vitro Transforming Growth Factor- $\beta 1$ Induced Fibroblast to Myofibroblast Transition: Involvement of Vascular Endothelial Growth Factor (VEGF)-A/VEGF Receptor1-Mediated Signaling. Cells. 2018 Sep; 7(9):E142.

Chellini F, Tani A, Zecchi-Orlandini S, Sassoli C. Influence of Platelet-Rich and Platelet-Poor Plasma on Endogenous Mechanisms of Skeletal Muscle Repair/Regeneration. Int J Mol Sci. 2019 Feb;20(3):E683

Cianforlini M, Mattioli-Belmonte M, Manzotti S, Chiurazzi E, Piani M, Orlando F, et al. Effect of platelet rich plasma concentration on skeletal muscle regeneration: an experimental study. J Biol Regul Homeost Agents. 2015 Oct-Dec;29(4 Suppl):47-55.

Delos D, Leineweber MJ, Chaudhury S, Alzoobaee S, Gao Y, Rodeo SA. The effect of platelet-rich plasma on muscle contusion healing in a rat model. Am J Sports Med. 2014 Sep;42(9):2067-74.

Duan X, Ji M, Deng F, Sun Z, Lin Z. Effects of connective tissue growth factor on human periodontal ligament fibroblasts. Arch Oral Biol. 2017 Dec;84:37-44.

Duryagina R, Thieme S, Anastassiadis K, Werner C, Schneider S, Wobus M, et al. Overexpression of Jagged-1 and its intracellular domain in human mesenchymal stromal cells differentially affect the interaction with hematopoietic stem and progenitor cells. Stem Cells Dev. 2013 Oct;22(20):2736-50.

El Agha E, Kramann R, Schneider RK, Li X, Seeger W, Humphreys BD, et al. Mesenchymal Stem Cells in Fibrotic Disease. Cell Stem Cell. 2017 Aug;21(2):166-77.

Fang F, Huang RL, Zheng Y, Liu M, Huo R. Bone marrow derived mesenchymal stem cells inhibit the proliferative and profibrotic phenotype of hypertrophic scar fibroblasts and keloid fibroblasts through paracrine signaling. J Dermatol Sci. 2016 Aug;83(2):95-105.

Fang L, Murphy AJ, Dart AM. A clinical perspective of antifibrotic therapies for cardiovascular disease. Front Pharmacol. 2017 Apr;8:186.

Ferro F, Spelat R, Shaw G, Duffy N, Islam MN, O'Shea PM, et al. Survival/adaptation of bone marrow-derived mesenchymal stem cells after long-term starvation through selective processes. Stem Cells. 2019 Jun;37(6):81327.

Formigli L, Benvenuti S, Mercatelli R, Quercioli F, Tani A, Mirabella C, et al. Dermal matrix scaffold engineered with adult mesenchymal stem cells and platelet-rich plasma as a potential tool for tissue repair and regeneration. J Tissue Eng Regen Med. 2012 Feb;6(2):12534. 
Formigli L, Paternostro F, Tani A, Mirabella C, Quattrini Li A, Nosi D, et al. MSCs seeded on bioengineered scaffolds improve skin wound healing in rats. Wound Repair Regen. 2015 Jan-Feb;23(1):115-23.

Galie PA, Stegemann JP. Injection of mesenchymal stromal cells into a mechanically stimulated in vitro model of cardiac fibrosis has paracrine effects on resident fibroblasts. $\mathrm{Cy}$ totherapy. 2014 Jul;16(7):906-14.

Gallego-Muñoz P, Ibares-Frías L, Valsero-Blanco MC, Cantalapiedra-Rodriguez R, MerayoLloves J, Martínez-García MC. Effects of TGF $\beta 1$, PDGF-BB, and bFGF, on human corneal fibroblasts proliferation and differentiation during stromal repair. Cytokine. 2017 Aug;96:94-101.

Guillodo Y, Madouas G, Simon T, Le Dauphin H, Saraux A. Platelet-rich plasma (PRP) treatment of sports-related severe acute hamstring injuries. Muscles Ligaments Tendons J. 2016 Feb;5(4):284-8.

Hoberman AR, Cirino C, McCarthy MB, Cote MP, Pauzenberger L, Beitzel K, et al. Bone Marrow-Derived Mesenchymal Stromal Cells Enhanced by Platelet-Rich Plasma Maintain Adhesion to Scaffolds in Arthroscopic Simulation. Arthroscopy. 2018 Mar;34(3):872-81.

Hoeferlin LA, Huynh QK, Mietla JA, Sell SA, Tucker J, Chalfant CE, et al. The lipid portion of activated platelet-rich plasma significantly contributes to its wound healing properties. Adv Wound Care (New Rochelle). 2015 Feb; 4(2):100-9.

Hosni Ahmed H, Rashed LA, Mahfouz S, Elsayed Hussein R, Alkaffas M, Mostafa S, et al. Can mesenchymal stem cells pretreated with platelet-rich plasma modulate tissue remodeling in a rat with burned skin? Biochem Cell Biol. 2017 Oct;95(5):537-48.

$\mathrm{Hu}$ C, Li L. Preconditioning influences mesenchymal stem cell properties in vitro and in vivo. J Cell Mol Med. 2018 Mar;22(3): $1428-42$.

Huang S, Wu Y, Gao D, Fu X. Paracrine action of mesenchymal stromal cells delivered by microspheres contributes to cutaneous wound healing and prevents scar formation in mice. Cytotherapy. 2015 Jul;17(7):922-31.

Jalowiec JM, D’Este M, Bara JJ, Denom J, Menzel $\mathrm{U}$, Alini $\mathrm{M}$, et al. An in vitro investigation of platelet-rich plasma-gel as a cell and growth factor delivery vehicle for tissue engineering. Tissue Eng Part C Methods. 2016 Jan;22(1): 49-58.

Jang HY, Myoung SM, Choe JM, Kim T, Cheon YP, Kim YM, et al. Effects of autologous platelet-rich plasma on regeneration of damaged endometrium in female rats. Yonsei Med J. 2017 Nov;58(6):1195-203.

Jang YO, Jun BG, Baik SK, Kim MY, Kwon SO. Inhibition of hepatic stellate cells by bone marrow-derived mesenchymal stem cells in hepatic fibrosis. Clin Mol Hepatol. 2015 Jun; 21(2):141-9.

Jun JI, Lau LF. Resolution of organ fibrosis. J Clin Invest. 2018 Jan;128(1):97-107.
Kelc R, Vogrin M. Concerns about fibrosis development after scaffolded PRP therapy of muscle injuries: commentary on an article by Sanchez et al.: "Muscle repair: platelet-rich plasma derivates as a bridge from spontaneity to intervention". Injury. $2015 \mathrm{Feb}$;46(2):428.

Kim M, Kim KH, Song SU, Yi TG, Yoon SH, Park $\mathrm{SR}$, et al. Transplantation of human bone marrow-derived clonal mesenchymal stem cells reduces fibrotic scar formation in a rat spinal cord injury model. J Tissue Eng Regen Med. 2018 Feb;12(2):e1034-45.

Lan YW, Choo KB, Chen CM, Hung TH, Chen YB, Hsieh $\mathrm{CH}$, et al. Hypoxia-preconditioned mesenchymal stem cells attenuate bleomycininduced pulmonary fibrosis. Stem Cell Res Ther. 2015 May;6(1):97.

Lei J, Chai Y, Xiao J, Hu H, Liu Z, Xiao Y, et al. Antifibrotic potential of bone marrow-derived mesenchymal stem cells in biliary atresia mice. Mol Med Rep. 2018 Oct;18(4): 3983-8.

Li C, Zheng S, You H, Liu X, Lin M, Yang L, et al. Sphingosine 1-phosphate (S1P)/S1P receptors are involved in human liver fibrosis by action on hepatic myofibroblasts motility. J Hepatol. 2011 Jun;54(6):1205-13.

Li H, Ghazanfari R, Zacharaki D, Lim HC, Scheding $\mathrm{S}$. Isolation and characterization of primary bone marrow mesenchymal stromal cells. Ann N Y Acad Sci. 2016 Apr;1370(1): 109-18.

Lim R, Ricardo SD, Sievert W. Cell-based therapies for tissue fibrosis. Front Pharmacol. 2017 Sep;8:633

Lunde IG, Herum KM, Carlson CC, Christensen G. Syndecans in heart fibrosis. Cell Tissue Res. 2016 Sep;365(3):539-52.

Lynch MD, Bashir S. Applications of platelet-rich plasma in dermatology: A critical appraisal of the literature. J Dermatolog Treat. 2016;27(3): 285-9.

Mahdy MA. Skeletal muscle fibrosis: an overview. Cell Tissue Res. 2019 Mar;375(3):575-88.

Manohar M, Kandikattu HK, Verma AK, Mishra A. IL-15 regulates fibrosis and inflammation in a mouse model of chronic pancreatitis. Am J Physiol Gastrointest Liver Physiol. 2018 Dec;315(6):G954-65.

Manon-Jensen T, Multhaupt HA, Couchman JR. Mapping of matrix metalloproteinase cleavage sites on syndecan- 1 and syndecan- 4 ectodomains. FEBS J. 2013 May;280(10):2320-31.

Miao C, Lei M, Hu W, Han S, Wang Q. A brief review: the therapeutic potential of bone marrow mesenchymal stem cells in myocardial infarction. Stem Cell Res Ther. 2017 Nov; $8(1): 242$.

Moghadam A, Khozani TT, Mafi A, Namavar MR, Dehghani F. Effects of platelet-rich plasma on kidney regeneration in gentamicin-induced nephrotoxicity. J Korean Med Sci. 2017 Jan;32(1):13-21.

Nistri S, Sassoli C, Bani D. Notch signaling in ischemic damage and fibrosis: evidence and clues from the heart. Front Pharmacol. 2017 Apr;8:187.
Pakshir P, Hinz B. The big five in fibrosis: macrophages, myofibroblasts, matrix, mechanics, and miscommunication. Matrix Biol. 2018 Aug;68-69:81-93.

Prockop DJ. Inflammation, fibrosis, and modulation of the process by mesenchymal stem/ stromal cells. Matrix Biol. 2016 Apr;51:7-13.

Qiao J, An N, Ouyang X. Quantification of growth factors in different platelet concentrates. Platelets. 2017 Dec;28(8):774-8.

Rabelink TJ, Little MH. Stromal cells in tissue homeostasis: balancing regeneration and fibrosis. Nat Rev Nephrol. 2013 Dec;9(12):747-53.

Reurink G, Goudswaard GJ, Moen MH, Weir A, Verhaar JA, Bierma-Zeinstra SM, et al.; Dutch HIT-study Investigators. Rationale, secondary outcome scores and 1-year follow-up of a randomised trial of platelet-rich plasma injections in acute hamstring muscle injury: the Dutch Hamstring Injection Therapy study. $\mathrm{Br}$ J Sports Med. 2015 Sep;49(18):1206-12.

Rosenbloom J, Macarak E, Piera-Velazquez S, Jimenez SA. Human fibrotic diseases: current challenges in fibrosis research. Methods $\mathrm{Mol}$ Biol. 2017;1627:1-23.

Royce SG, Shen M, Patel KP, Huuskes BM, Ricardo SD, Samuel CS. Mesenchymal stem cells and serelaxin synergistically abrogate established airway fibrosis in an experimental model of chronic allergic airways disease. Stem Cell Res (Amst). 2015 Nov;15(3):495-505.

Rozier P, Maria A, Goulabchand R, Jorgensen C, Guilpain P, Noël D. Mesenchymal stem cells in systemic sclerosis: allogenic or autologous approaches for therapeutic use? Front Immunol. 2018 Dec 14;9:2938.

Rubio-Azpeitia E, Andia I. Partnership between platelet-rich plasma and mesenchymal stem cells: in vitro experience. Muscles Ligaments Tendons J. 2014 May;4(1):52-62.

Saberi K, Pasbakhsh P, Omidi A, BorhaniHaghighi M, Nekoonam S, Omidi N, et al. Melatonin preconditioning of bone marrowderived mesenchymal stem cells promotes their engraftment and improves renal regeneration in a rat model of chronic kidney disease. J Mol Histol. 2019 Apr;50(2):129-40.

Sanchez-Avila RM, Merayo-Lloves J, Riestra AC, Berisa S, Lisa C, Sánchez JA, et al. Plasma rich in growth factors membrane as coadjuvant treatment in the surgery of ocular surface disorders. Medicine (Baltimore). 2018 Apr;97(17):e0242.

Sassoli C, Chellini F, Pini A, Tani A, Nistri S, Nosi $D$, et al. Relaxin prevents cardiac fibroblastmyofibroblast transition via notch-1-mediated inhibition of TGF- $\beta / S \operatorname{mad} 3$ signaling. PLoS One. 2013 May;8(5):e63896.

Sassoli C, Frati A, Tani A, Anderloni G, Pierucci F, Matteini F, et al. Mesenchymal stromal cell secreted sphingosine 1-phosphate (S1P) exerts a stimulatory effect on skeletal myoblast proliferation. PLoS One. 2014a Sep;9(9):e108662.

Sassoli C, Nosi D, Tani A, Chellini F, Mazzanti B, Quercioli F, et al. Defining the role of mesenchymal stromal cells on the regulation of matrix metalloproteinases in skeletal muscle cells. Exp Cell Res. 2014b May;323(2):297-313. 
Sassoli C, Pierucci F, Tani A, Frati A, Chellini F, Matteini F, et al. Sphingosine 1-phosphate receptor 1 is required for MMP-2 function in bone marrow mesenchymal stromal cells: implications for cytoskeleton assembly and proliferation. Stem Cells Int. 2018a Mar;2018: 5034679.

Sassoli C, Pini A, Chellini F, Mazzanti B, Nistri S, Nosi D, et al. Bone marrow mesenchymal stromal cells stimulate skeletal myoblast proliferation through the paracrine release of VEGF. PLoS One. 2012;7(7):e37512.

Sassoli C, Pini A, Mazzanti B, Quercioli F, Nistri $S$, Saccardi R, et al. Mesenchymal stromal cells affect cardiomyocyte growth through juxtacrine Notch-1/Jagged-1 signaling and paracrine mechanisms: clues for cardiac regeneration. J Mol Cell Cardiol. 2011 Sep;51(3):399408.

Sassoli C, Vallone L, Tani A, Chellini F, Nosi D, Zecchi-Orlandini S. Combined use of bone marrow-derived mesenchymal stromal cells (BM-MSCs) and platelet rich plasma (PRP) stimulates proliferation and differentiation of myoblasts in vitro: new therapeutic perspectives for skeletal muscle repair/regeneration. Cell Tissue Res. 2018b Jun;372(3):549-70.

Sayadi LR, Obagi Z, Banyard DA, Ziegler ME, Prussak J, Tomlinson L, et al. Platelet-rich plasma, adipose tissue, and scar modulation. Aesthet Surg J. 2018 Nov;38(12):1351-62.

Schroeder CC, Scariot JS, Ribeiro JC, Deliberador TM, Giovanini AM. Platelet rich plasma (PRP) produces an atherofibrotic histophenotype during craniofacial bone repair due to changes of immunohistochemical expression of erk $1 / 2, \mathrm{p} 38 \alpha / \beta$, adiponectin and elevated presence of cells exhibiting b-scavenger receptor (CD36+). Braz Dent J. 2016 May-Jun; 27(3):243-54.
Shoeib HM, Keshk WA, Foda AM, Abo El Noeman SE. A study on the regenerative effect of platelet-rich plasma on experimentally induced hepatic damage in albino rats. Can J Physiol Pharmacol. 2018 Jun;96(6):630-6.

Squillaro T, Peluso G, Galderisi U. Clinical Trials With Mesenchymal Stem Cells: an Update. Cell Transplant. 2016;25(5):829-48.

Srour N, Thébaud B. Mesenchymal Stromal Cells in Animal Bleomycin Pulmonary Fibrosis Models: A Systematic Review. Stem Cells Transl Med. 2015 Dec;4(12):1500-10.

Tani A, Chellini F, Giannelli M, Nosi D, ZecchiOrlandini S, Sassoli C. Red (635 nm), NearInfrared $(808 \mathrm{~nm})$ and Violet-Blue $(405 \mathrm{~nm})$ Photobiomodulation Potentiality on Human Osteoblasts and Mesenchymal Stromal Cells: A Morphological and Molecular In Vitro Study. Int J Mol Sci. 2018 Jul;19(7):E1946.

Tavukcu HH, Aytaç Ö, Atuğ F, Alev B, Çevik Ö, Bülbül N, et al. Protective effect of platelet-rich plasma on urethral injury model of male rats. Neurourol Urodyn. 2018 Apr;37(4):1286-93.

Vestri A, Pierucci F, Frati A, Monaco L, Meacci E. Sphingosine 1-phosphate receptors: do they have a therapeutic potential in cardiac fibrosis? Front Pharmacol. 2017 Jun;8:296.

Vu TD, Pal SN, Ti LK, Martinez EC, Rufaihah AJ, Ling $\mathrm{LH}$, et al. An autologous platelet-rich plasma hydrogel compound restores left ventricular structure, function and ameliorates adverse remodeling in a minimally invasive large animal myocardial restoration model: a translational approach: Vu and Pal "Myocardial Repair: PRP, Hydrogel and Supplements". Biomaterials. 2015 Mar;45:27-35.

Walraven M, Hinz B. Therapeutic approaches to control tissue repair and fibrosis: extracellular matrix as a game changer. Matrix Biol. 2018 Oct;71-72:205-24.

Weiskirchen R, Weiskirchen S, Tacke F. Organ and tissue fibrosis: molecular signals, cellular mechanisms and translational implications. Mol Aspects Med. 2019 Feb;65:2-15.
Xian LJ, Chowdhury SR, Bin Saim A, Idrus RB. Concentration-dependent effect of plateletrich plasma on keratinocyte and fibroblast wound healing. Cytotherapy. 2015 Mar;17(3): 293-300.

Xing L, Song E, Yu CY, Jia XB, Ma J, Sui MS, et al. Bone marrow-derived mesenchymal stem cells attenuate tubulointerstitial injury through multiple mechanisms in UUO model. J Cell Biochem. 2019;120(6):9737-46.

Xu B, Luo Y, Liu Y, Li BY, Wang Y. Platelet-derived growth factor-BB enhances MSC-mediated cardioprotection via suppression of miR320 expression. Am J Physiol Heart Circ Physiol. 2015 May;308(9):H980-9.

Yang Y, Liu Z, Wang J, Chai Y, Su J, Shi F, et al. The effect of interferon gamma on conventional fractionated radiation-induced damage and fibrosis in the pelvic tissue of rabbits. Ther Clin Risk Manag. 2016 May;9:755-62.

Yoon S, Kang G, Eom GH. HDAC Inhibitors: Therapeutic Potential in Fibrosis-Associated Human Diseases. Int J Mol Sci. 2019 Mar; 20(6):E1329.

Zhao F, Liu W, Yue S, Yang L, Hua Q, Zhou Y, et al. Pretreatment with G-CSF could enhance the antifibrotic effect of BM-MSCs on pulmonary fibrosis. Stem Cells Int. 2019a Jan;2019: 1726743.

Zhao T, Sun F, Liu J, Ding T, She J, Mao F, et al. Emerging role of mesenchymal stem cell-derived exosomes in regenerative medicine. Curr Stem Cell Res Ther. 2019b. DOI: 10.217 4/1574888X14666190228103230.; $\quad$ Epub ahead of print.

Zuccaro J, Ziolkowski N, Fish J. A Systematic Review of the Effectiveness of Laser Therapy for Hypertrophic Burn Scars. Clin Plast Surg. 2017 Oct;44(4):767-79. 